Aus dem Anatomischen Institut der Med. Fakultät, Univ. Okayama

(Vorstand: Prof. M. SEKI).

\title{
Elektronoskopische Beobachtung der mit hochfrequentem Schall behandelten fixierten Erythrocyten und Bestimmung ihrer Ultrastrukturdichte auf färberischem Wege.
}

\author{
超音波を作用させた固定赤血球の電子鏡観察と染色法による \\ その超構密度の測定.
}

Hakushi YASUDA 妿田博志。

[Eingegangen am 7. September 1954.]

Schon früher haben WOOD und LOOMIS (1927) die Zerstörung der Erythrocyten des Menschen durch die Ultraschallwellen festgestellt. HARVEY und LOOMIS (1928) und JOHNSON (1929) beobachteten auch die dadurch verursachten Zerstörungen der menschichen Erythrocyten und Protozoen. NAGASAWA (1942) sah, daß bei den kernhaltigen Erythrocyten der Kern nach der Zellmembran wanderte, wiederholt sich daran stieß und schließlich durch einen Bruch hinausging.

Der Verfasser hat die fixierten Erythrocyten nach der Einwirkung der Ultraschallwellen im Elektronoskop untersucht und hat andererseits die Veränderungen der ultrastrukturelle Dichte der Zellen mit Hilfe der sog. Azanfärbung bestimmt.

\section{Material und Methode.}

Kernlose Erythrocyten des erwachsenen Kaninchens und Kernhaltige des Haushuhns wurden zu diesen Versuch herangezogen. Man nahm das Blut aus der Ohrvene des Kaninchens bzw. der Flügelvene des Haushuhns, strich es auf ein rundliches Deckglas von etwa $1 \mathrm{~cm}$ Durchmesser aus, setzte es, ehe es zur Trocknung kam, dem Dampf aus der $1 \%$ gen Osmiumsäure drei Minuten lang aus und brachte es schließlich in destilliertes Wasser. Nach etwa 1 Stunde schüttelte man das Wasser leicht, löste das ausgestrichene Blut vom Deckglas ab und tat es samt dem Wasser in ein hartes Pyrexglasröhrchen ron $20 \mathrm{~cm}$ Länge, $1.5 \mathrm{~cm}$ Kaliber und etwa $0.5 \mathrm{~mm}$ Wanddicke. Das Glasröhrchen wurde so gestellt, daß seine Kuppe sich mit der Ölfläche im Beschallungsgerät berührt. Man hielt es immer in der Mitte des hervorspingenden Öles und ließ die Ulltraschallwellen zu Wirkung kommen. Die Erythrocyten wurden dann einerseits im Elektronoskop untersucht und andererseits nach der HEIDENHAINschen Azan- sowie nach der GIEMSA- 
methode gefärbt. Um die Färbbarkeiten der Erythrocyten nach den Wirkung der Ultraschallwellen von verschiedener Stärke genau zu vergleichen, färbte man eine Reihe von Präparaten gleichzeitig und behandelte sie auch gleichzeitig nach.

Das Beschallungsgerät war der piezoelektrische S-200 Typus von SHIMAZU mit Bergkristallen als Schwingungszeuger. Der Schwingungskreis des Gerätes gehört zu dem HARTLEYschen; seine Schwingungsvakuumröhre ist das U. V. 204 A von TOSHIBA. Bei unseren Versuchen wurden die Erythrocyten im Gerät unter dem einführenden $150 \mathrm{~mA}$ Strom aus $1500 \mathrm{~V}$ Spannung mit den Wellen von $710 \mathrm{kc}$ Frequenz 140 Minuten lang behandelt.

\section{Ergebnisse.}

\section{A. An den kernlosen Erythrocyten des Kaninchens.}

1. Bild von der Beschallung.

Die Abb. 1 stellt das Elektronbild des normalen Erythrocyten dar, der mit Osmiumsäuredampf fixiert und im destillierten Wasser gehalten worden war. Er ist gleichmäßig schattendicht.

Nach der Azanfärbung erscheint er orange (Abb. 20a). Das verrät uns eine hohe Ultrastrukturdichte des Erythrocyten. Nach der GIEMSAfärbung färbt er sich hellrot (Abb. 21a).

2. Nach Beschallung von 1 Minute.

Die Erythrocyten zeigen im Elektronoskop noch keine Veränderung. Nach der Azanfärbung wird der zentrale Teil der Zellen etwas heller, aber die Zellen färben sich immer noch orange (Abb. 20b).

Nach der GIEMSAfärbung erkennt man auch eine leichte Helligkeit des zentralen Teils der Zellen (Abb. 21b). Dieser Teil ist der Sitz des von ISHIMARU (1953) in den Erythrocyten von allen Säugetieren gefundenen nukleoiden Körpers.

3. Nach Beschallung von 2 Minuten.

Wie es in Abb. 2 dargestellt ist, läßt die Mitte des Erythrocyten den Elektronenstrahl hindurch, ist aber noch nicht gebrochen. Nach der Azanfärbuug ist die Randzone orange und die Mitte rötlichorange färbbar (Abb. 20c u. 20d).

Nach der GIEMSAfärbung erscheint auch die Mitte des Erythrocyten heller (Abb. 21c). Die Farbnuance ist aber fast dies̀elbe wie vorher.

4. Nach Beschallung von 3 Minuten langer.

Die Erythrocyten mit einem rundlichen Bruch in ihrer Mitte sind mit solchen, deren Mitte noch nicht gebrochen, aber schattenschwach ist, vermengt vorhanden (Abb. 3).

Nach der Azanmethode wird die Umgebung des Bruchs mit einem 
stärkeren rötlichen Ton gefärbt, der übrige Hauptteil aber fast wie vorher (Abb. 20e). Die Färbbarkeit der Erythrocyten nach der GIEMSAmethode bleibt unverändeṛt (Abb. 21d).

5. Nach Beschallung von 4 Minuten.

Die meisten Erythrocyten werden in der Mitte durchlöchert (Abb. $4)$.

Die Etythrocyten werden nach der Azanfärbung mit einem stärkeren rötlichen Ton gefärbt als vorher und erscheinen gelblich rot (Abb. 20f). Ihre Struktur ist also aufgelockert.

Auch nach GIEMSA sind die Erythrocyten nunmehr purpurn anfärbbar (Abb. 21e).

6. Nach Beschallung von 5-10 Minuten.

Wie in Abb. 5 gezeigt, vergrößert sich das zentrale Loch der Erythrocyten. Die Kontur der Zellen wird auch häufig verändert (Abb. 6).

Nach der Azanmethode färben sich die Erythrocyten mit zunehmendem rötlichem Ton (Abb. 20g u. 20h), und nach GIEMSA mit zunehmendem violetten Ton (Abb. 21f).

7. Nach Beschallung von 15 Minuten.

Von dem zentralen Loch der Erythrocyten ausgehend geht ein Riß nach außen, und der Erythrocytenring wird schließlich gespalten (Abb. 7). Der dem Riß benachbarte Teil des Erythrocyten ist etwas schattenschwächer.

Die Färbbarkeit der Zellen nach der Azan- und GIEMSAfärbung ist die gleiche wie vorher.

8. Nach Beschallung von mehr als 20 Minuten.

Die Abb. 8, 9, 10 und 11 stellen die 20, 25, 30 bzw. 40 Minuten lang beschallten Erythrocyten dar. Die Zellen sind mehr oder weniger stark gebrochen, daneben sind oft reichlich Zelltrümener zu sehen.

Die gebrochenen Erythrocyten färben sich nun nach der Azanmethode violettrot (Abb. 20i). Wie man es in Abb. 20j sieht, wird das gebrochene Ende der Erythrocytenstücke oft deutlich blau gefärbt. Mit der Verlängerung der Beschallungszeit werden die Bruchstückc immer stärker blau färbbar (Abb. 20k u. 201). Das bedeutet, daß ihre Ultrastrukturdichte sich immer mehr vermindert.

Auch nach der GIEMSAfärbung werden sie immer stärker blau färbbar und schließlich violettblau gefärbt (Abb. 21g).

\section{B. An den kernhaltigen Erythrocyten des Haushuhns.}

1. Bild von der Beschallung.

In Abb. 12 ist ein mit dem Osmiumsäuredampf fixierter Erythrocyt des Haushuhns ohne Beschallung gezeigt.

Nach der Azanmethode werden die Erythrocyten, einschließlich des 
Kerns, gleichmäßig orange gefärbt (Abb. 22a). Sie färben sich nach GIEMSA schwach rot mit violetter Nuance (Abb. 23a).

2. Nach Beschallung von 1-2 Minuten.

Noch ist keine morphologische Veränderung in der Elektronographie bemerkbar.

Die Grenze zwischen dem Cytoplasma und dem Kern erscheint gelegentlich nach der Azanfärbung rötlichorange (Abb. 22b u. 22c). Nach der GIEMSAfärbung läßt sich noch keine Veränderung erkennen.

3. Nach Beschallung von 3 Minuten.

Es zeigt sich noch keine morphologische Veränderung der Erythrocyten, aber der Zellkern färbt sich mit stärkerer rötlicher Nuance nach der Azanfärbung (Abb. 22e).

Mit GIEMSA färben sich ganze Kerne violett (Abb. 23b).

4. Nach Beschallung von 4 Minuten.

Wie in Abb. 3 dargestellt, bemerkt man hier erstmalig ein Löchlein in der Mitte der Erythrocyten.

Nach der Azanfärbung wird der Zellkern immer noch stärker rot gefärbt (Abb. 22f). Nach der GIEMSAfärbung erscheint die ganze Zelle stärker violett (Abb. 23c).

5. Nach Beschallung von 5-10 Minuten.

Das zentrale Loch der Erythrocyten wird größer (Abb. 14). Der Kern färbt sich nach der Azanfärbung noch stärker rot als vorher (Abb. $22 \mathrm{~g}$ ). In seinem Loch werden gelegentlich nach der GIEMSAfärbung blaßblau färbbare Trümmer gesehen (Abb. 23d).

6. Nach Beschallung von 10--20 Minuten.

Die kleine Masse neben dem Erythrocyten in Abb. 15 ist ein aus einem Erythrocytenleib herausgekommener Kern. Um das zentrale Loch des Erythrocyten in Abb. 16 ist die Zellsubstanz etwas schattenschwächer.

Die Abb. 22h, 22i, 22j und 22k zeigen Erythrocyten mit einem gebrochenen Kern in verschiedenem Grade nach der Azanfärbung. Die Reste des Kerns färben sich rot. Um das Loch kann auch ein Teil des Zelleibes rötlich angefärbt werden. Der Zelleib wird nach GIEMSA violett mit verstärktem blauen Ton gefärbt (Abb. 23e).

7. Nach Beschallung von mehr als 20 Minuten.

Die Abb. 17, 18 und 19 veranschaulichen gebrochene Erythrocytenstücke nach $20,30 \mathrm{bzw}$. 40 Minuten.

In Abb. 221, 22m, 22n und 22o sieht man nach der Azanmethode gefärbte Erythrocyten. Der Zelleib färbt sich teilweise mehr oder weniger stark orangerot bis rot. Nach mehr als 30 Minuten wird der Zelleib um die Durchlöcherung und am Ende der Abbrechung violett bis blau anfärbbar (Abb. 22p, $22 q$ u. 22r). Nach 40 Minuten sieht man nur selten die Erythrocyten mit gebliebenen Kern. Ihr Kern tingiert 
sich stark blau, wobei der Zelleib bläulichorange erscheint (Abb. 22s u. 22t). Man ersieht daraus, daß durch die Beschallung die Ultrastruktur des Kerns wie auch des Zelleibes aufgelockert wird.

Die Erythrocyten färben sich nach GIEMSA bläulichviolett wie vorher (Abb. 23f u. 23g).

\section{Auswertung.}

Wenn man die Ultraschallwellen auf die fixierten kernlosen Erythrocyten des Kaninchens einwirken läßt, so wird die Mitte der Erythrocytenscheibe, der Sitz des nukleoiden Körper von ISHIMARU (1953), im Elektronoskop schattenschwächer und schließlich durchlöchert. Dieses Loch vergrößert sich nach ihrer Umgebung, und wenn sein Durchmeser mehr als ein Drittel von demjenigen des Zelleibes erreicht, so geht ein Riß aus dem Loch nach außen bis zum Erythrocytenrand. Bei den fixierten kernhaltigen Erythrocyten des Haushuhns entsteht auch ein Loch in der Mitte der Zelle, welches sich mehr und mehr vergrößert. Der Kern wird dann losgerissen. Die Erythrocyten ohne Kern teilen sich darauf in Stücke. Die Erscheinung, daß durch die Wellenwirkung die Mitte der Erythrocyten vorerst durchlöchert wird, deutet darauf hin, daß dort der Widerstand am schwächsten ist. In Übereinstimmung mit diesen Befunden hat SUGIOKA (1952) an den durch eine hypotonische Flüssigkeit hämolysierten Erythrocyten von Säugetieren eine schattenschwächere Portion vom 1.7-2.3 $\mu$ Durchmesser gesehen. $\mathrm{Zu}$ bemerken ist, daß an den nicht beschallten normalen Etythrocyten auf färberischem Wege noch kein Ort mit wenigerer Ultrastrukturdichte nachgewiesen wurde.

Die normalen Erythrocyten sind bei der Azanfärbung vornehmlich mit dem kleinmolekularen Farbstoff orange zu färben, aber werden durch die Wirkung der Ultraschallwellen mit immer größer molekularen Farbstoffen rotorange, rot, violettrot und schließlich violett färbbar. Die Ultrastruktur der Zellen wird also durch die Beschallung aufgelockert.

\section{Zusammenfassung.}

1. Durch die Einwirkung der Ultraschallwellen werden die mit dem Osmiumsäuredampf fixierten Erythrocyten des Kaninchens und Haushuhns vorerst in der Mitte durchlöchert und zerfallen dann in Stücke.

2. Durch die Färbung der Erythrocyten mit Farbstoffen vershiedener Dispersität ist festgestellt, daß die Beschallung nicht nur den groben Bau der Zellen zerstört, sondern auch ihre feinsten Strukturen aus Molekülen auflockert. 


\section{内 容 自 抄。}

固定せられた家忽の無核赤血球と鴊の有核赤血球に超音波を作用させ， その破壊状態を電子鏡検した。作用時間の経過と共に, 常に先ず血球の中 央部が毀れて孔が生じ，次に胞体に割目が出来て分解し，赤血球の中央部 の抵抗の最も弱いことが知られた。

他方 3 種の分散度と色の違う染料を用いるアッ⿻ン染色とより超音波作 用による赤血球の超構密度の変化が観察された。赤血球は橙より赤橙，赤， 紫赤，紫，遂に青に染るようになる。即ち次第に大分子の染料によく染る ようとなり，赤血球の分子で組立てられた最も微組の構造も次第飞疎化す ることが証明せられた。

\section{Literatur.}

Harvey, E. N. a. A. L. Loomis: High frequency sound waves of small intensity and their biological effects. Nature. 121 (1928). - Ishimaru, S., T. Matsuda, K. Terahata a. T. Shunto: Elektron-microscopical study on the nucleoid-body in every erythrocyte of mammals. (Jap.) Kaibo. Z. 28 (1953). Suppl. - Johnson, C. H. : The lethal effects of ultrasonic radiation. J. Physiol. 67 (1829). - Nagasawa S. : Über den hämolytische Mechanismus der Ultraschallwellen. (Jap.) Osaka I. Z. 41 (1942). - Sugioka, Z. : Electron microscopy of erythrocytes of various animals. (Jap. m. engl. Zfass.) HandaiIsi. 5 (1952). - Wood, R. W. a. A. L. Loomis: The physical effects of high frequency souud-waves of great intensity. Phil. Mag. 4 (1927).

\section{Erklärung der Tafelabbildungen.}

Abb. 1-19 sind Elektronbilder der mit dem Osmiumsäuredampf fixierten Erythrocyten. Die Erythrocyten in Abb. 20 und 22 sind nach der Azanmethode gefärbt, diejenigen in Abb. 21 und 23 aber nach der GIEMSAschen Methode. Die Photographien in der oberen Reihe der Abb. 20-23 sind von den farbigen Urbildern der Erythrocyten. In der unteren Reihe der Abbildungen sind die Farbe und die Stärke derselben mit Punkten und schrägen Linien ausgedrückt, derart, daß die Dichtigkeit der Punkte die Stärke des Oranges und die der schrägen Linien die Stärke des Blaues bzw. Violettes bedeuten, während die unberührten Bezirke sich fast rein rot färben.

Abb. 1. Normaler Erythrocyt des Kaninchens im Elektronoskop. $4000 \times$.

Abb. 2. Erythrocyt des Kaninchens. 2 Minuten lange Beschallung. $4000 \times$.

Abb. 3. Derselbe. 3 Minuten lange Beschallung. $2800 \times$.

Abb. 4. Derselbe. 4 Minuten lange Beschallung. $2800 \times$.

Abb. 5. Derselbe. 5 Minuten lange Beschallung. $4000 \times$.

Abb. 6. Derselbe. 8 Minuten lange Beschallung. $4000 \times$.

Abb. 7. Derselbe. 15 Minuten lange Beschallung. 2800×. 
Abb. 8. Derselbe. 20 Minuten lange Beschallung. $4000 \times$. Abb. 9. Derselbe. 25 Minuten lange Beschallung. $4000 \times$. Abb. 10. Derselbe. 30 Minuten lange Beschallung. $4000 \times$. Abb. 11. Derselbe. 40 Minuten lange Beschallung. $2800 \times$.

Abb. 12. Normaler Erythrocyt des Haushuhns im Elektronoskop. $2800 \times$.

Abb. 13. Erythrocyt des Haushuhns. 4 Minuten lange Beschallung. 2800× .

Abb. 14. Derselbe. 7 Minuten lange Beschallung. 2800 $\times$.

Abb. 15. Derselbe. 10 Minuten lange Beschallung. $2800 \times$.

Abb. 16. Derselbe. 15 Minuten lange Beschallung. $4000 \times$.

Abb. 17. Derselbe. 20 Minuten lange Beschallung. $4000 \times$.

Abb. 18. Derselbe. 30 Minuten lange Beschallung. $4000 \times$.

Abb. 19. Derselbe. 40 Minuten lange Beschallung. $4000 \times$.

Abb. 20. Erythrocyten des Kaninchens, gefärbt nach der Azanmethode. a nicht beschallt, b 1 Minute lang beschallt, c $u$. d 2 Minuten lang beschallt, e 3 Minuten lang beschallt, f 4 Minuten lang beschallt, g u. h 5-10 Minuten lang beschallt, i, j, $\mathbf{k}$ u. I mehr als 20 Minuten lang beschallt.

Abb. 21. Erythrocyten des Kaninchens, gefärbt nach der GIEMSAschen Methode. a nicht beschallt, b 1 Minute lang beschallt, c 2 Minuten lang beschallt, d 3 Minuten lang beschallt, e 4 Minuten lang beschallt, 5-10 Minuten lang beschallt, g mehr als 20 Minuten beschallt.

Abb. 22. Erythrocyten des Haushuhns, gefärbt nach der Azanmethode. a nicht beschallt, b u. c 1-2 Minuten lang beschallt, d u. e 3 Minuten lang beschallt, f 4 Minuten lang beschallt, $\mathbf{g}$ 5-10 Minuten lang beschallt, $\mathbf{h , ~} \mathbf{i}$, j, u. k 10-20 Minuten lang beschallt, I, $\mathbf{m}, \mathbf{n}$ u. o 20 Minuten lang beschallt, p, q u. $\mathbf{r} 30$ Minuten lang beschallt, s u. t 40 Minuten lang beschallt.

Abb. 23. Erythrocyten des Haushuhns, gefärbt nach der GIEMSAschen Methode. a nicht beschallt, b 3 Minuten lang beschallt, c 4 Minuten lang beschallt, d 5-10 Minuten lang beschallt, e 10-20 Minuten lang beschallt, f u. g mehr als 20 Minuten lang beschallt. 
H. YASUDA (1)

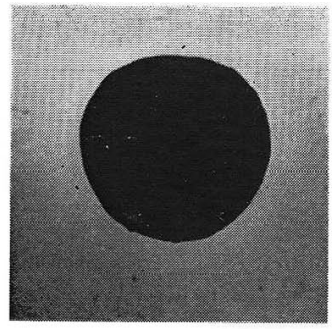

1

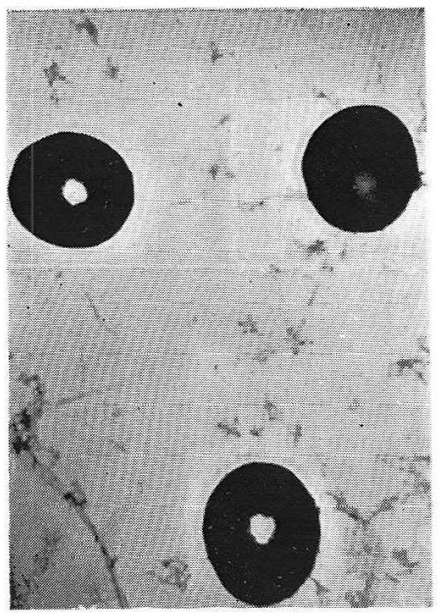

3

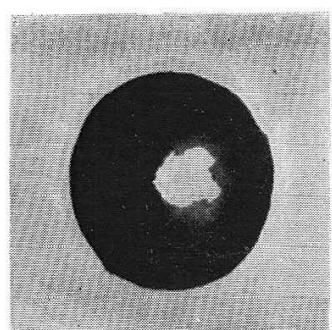

5

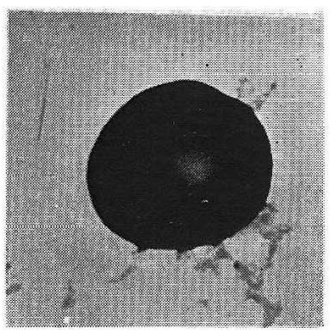

2
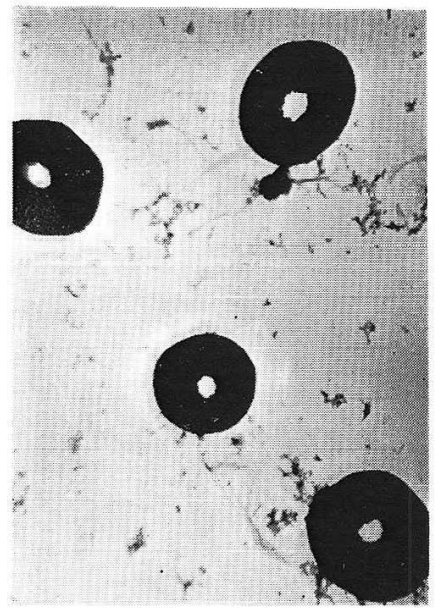

4

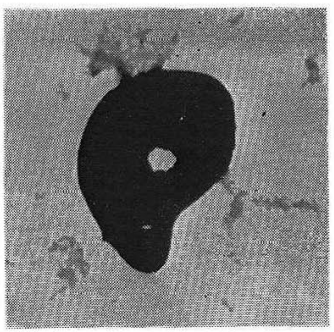

6 


\section{H. YASUDA (2)}
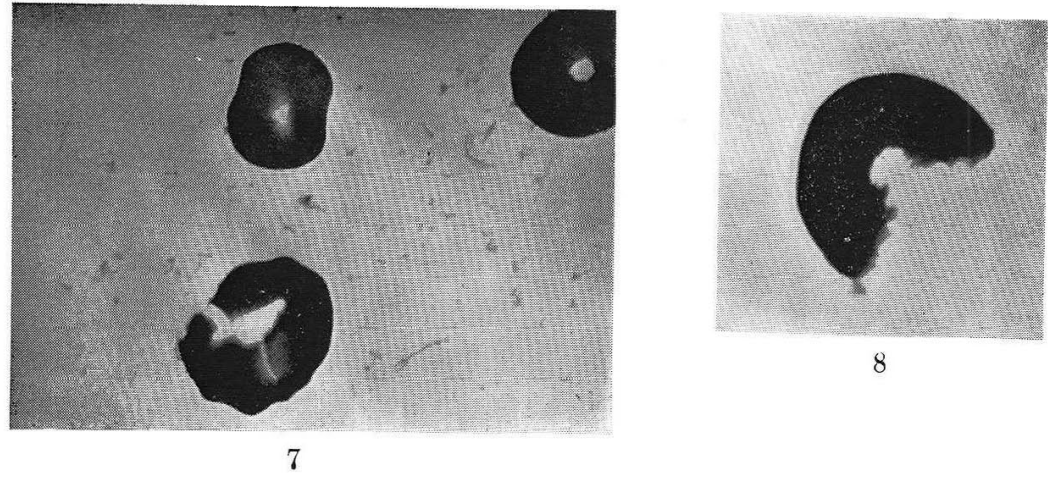

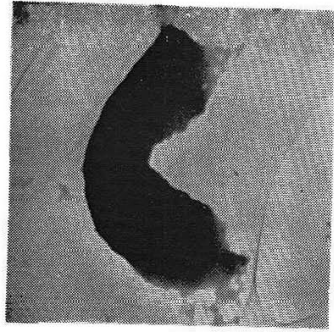

9

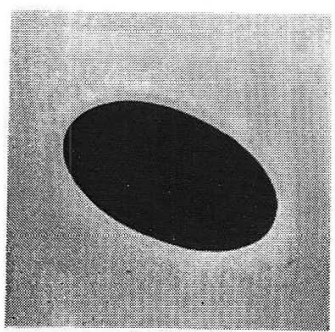

12

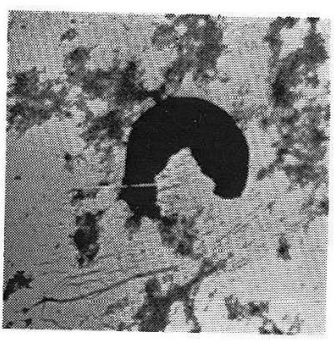

10

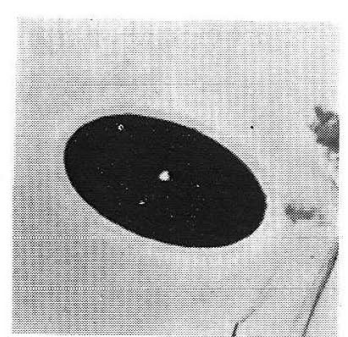

13

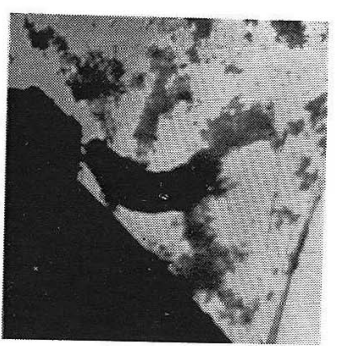

11

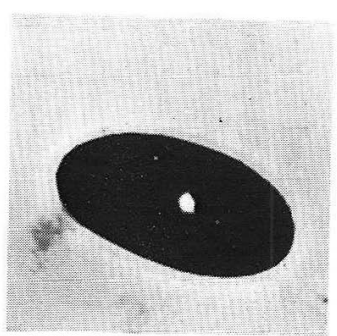

14 


\section{H. YASUDA (3)}

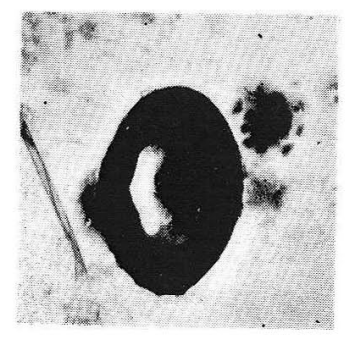

15

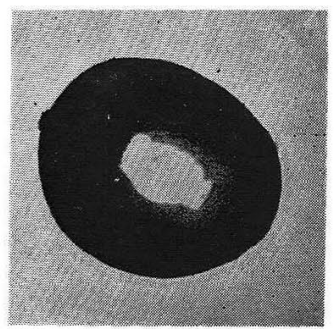

16

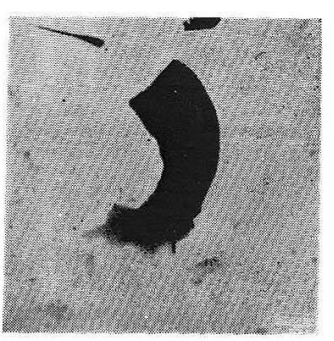

17

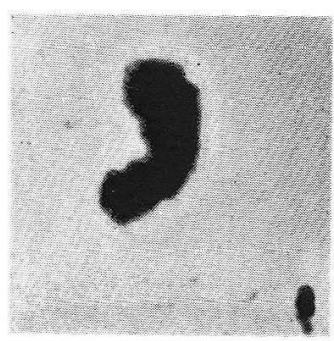

18

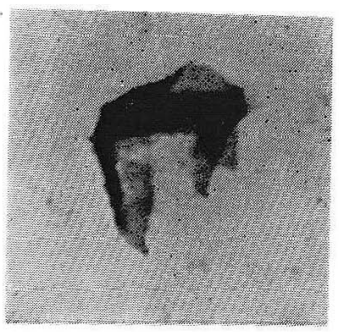

19
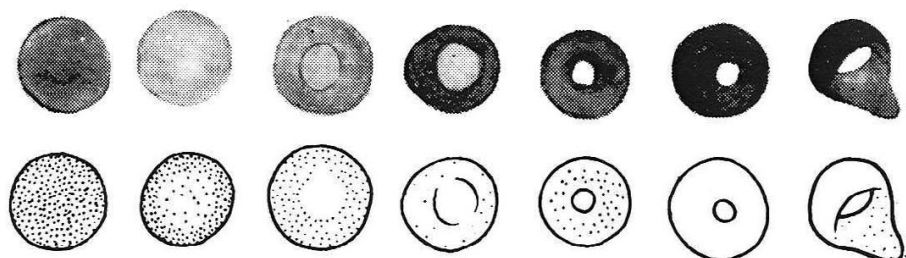

b
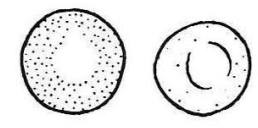

d
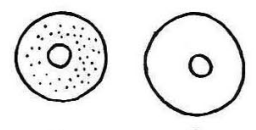

e

20
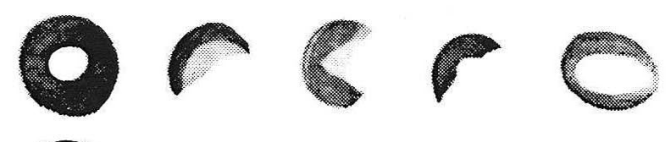

(0)

$\mathrm{h}$

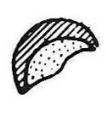

i
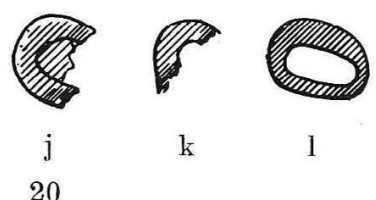

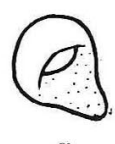

g 


\section{H. YASUDA (4)}
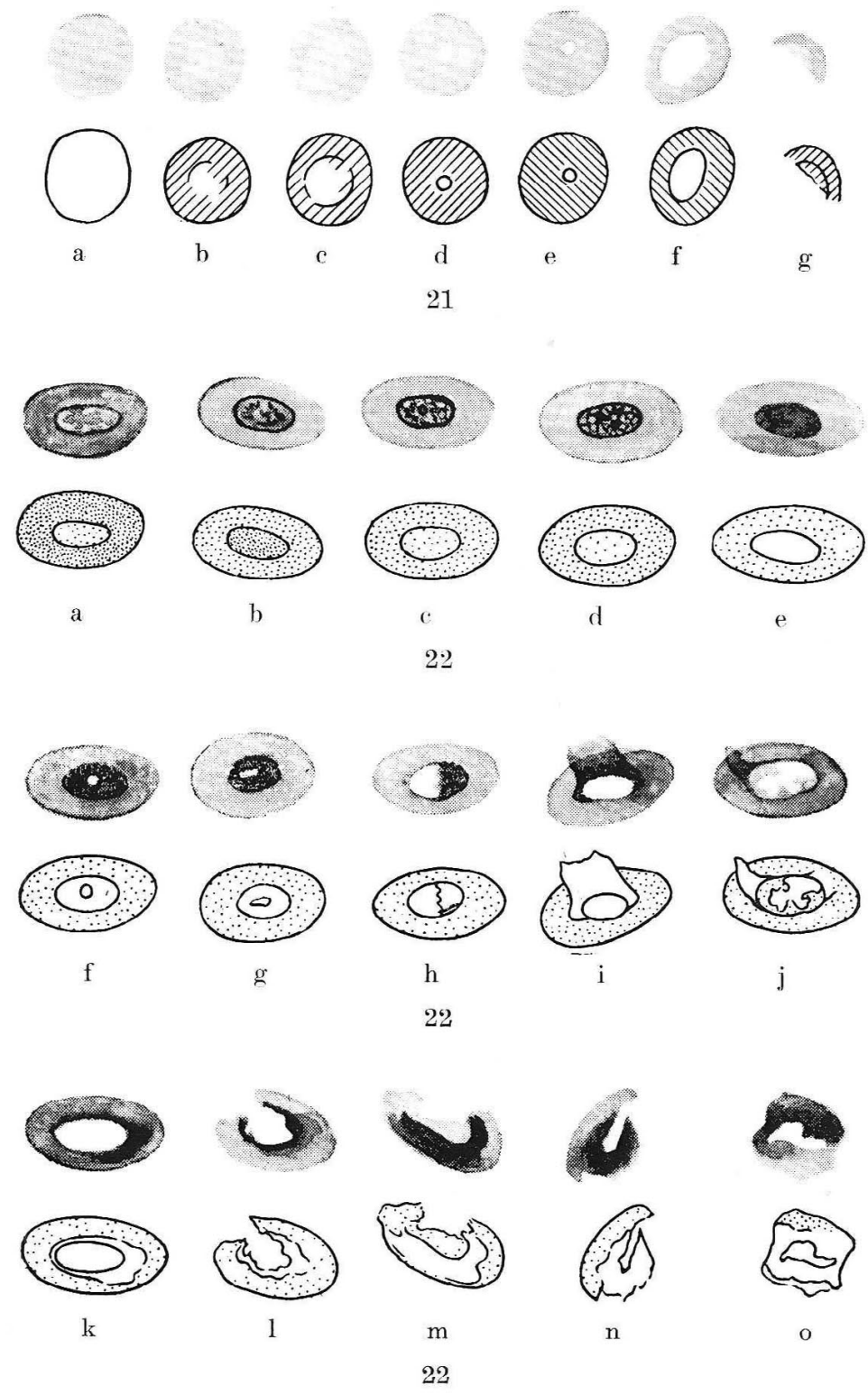
H. YASUDA ( 5 )
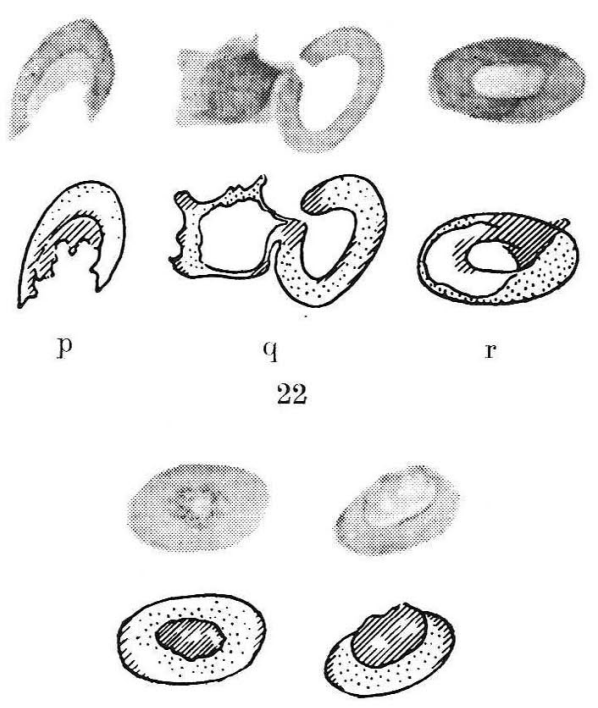

S

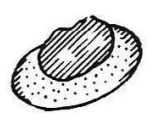

22

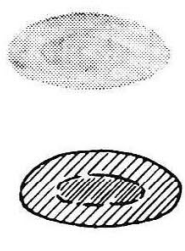

b

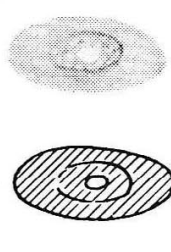

c
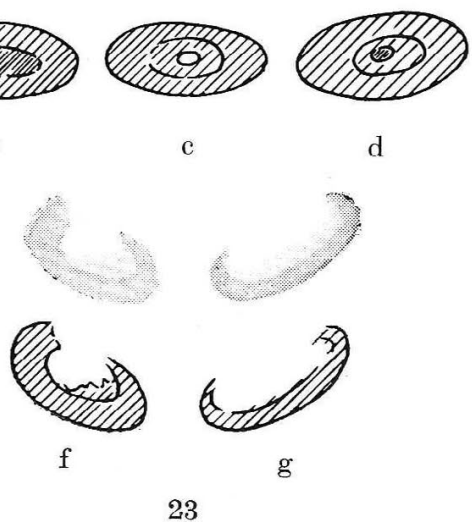

23

367

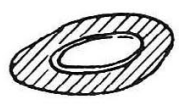

e 